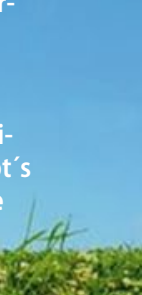

\title{
Alkohol ist nicht der Wohltäter
}

Epidemiologische Studien legen nahe, dass moderater Alkoholkonsum die kardiovaskuläre Sterblichkeit und Diabetesinzidenz senkt. Ob dies dem Alkohol oder den nicht alkoholischen Bestandteilen des Rotweins zuzuschreiben ist, ist nicht endgültig geklärt. Daher wurden die Einflüsse eines moderaten Rotweinkonsums auf Zucker- und Lipidstoffwechsel mit denen alkohol-

Chiva-Blanch G, Urpi-Sarda M,

Ros E et al. Clin Nutr 2012 Sep 3. pii: S0261-5614(12)00189-6. [Epub ahead of print] freien Rotweins und Gins bei 67 Männern mit hohem kardiovaskulärem Risiko geprüft. Nach der Run-in-Phase erhielten alle jeweils über 4 Wochen in verschiedener Folge alkoholfreien Rotwein oder normalen Rotwein oder Gin (jew. 30 g Alkohol/d). Vor und nach jeder Phase wurden nüchtern Glukose und Insulin, HOMA-Index, Lipo-, Apoproteine (ALP) und Adipokine bestimmt.

Ergebnisse: Die Glukose blieb unbeeinflusst. Dagegen sanken Insulinspiegel und HOMA-Index nach Rotwein mit oder ohne Alkohol. HDL, ALP A-I und -II stiegen nach Rotwein und Gin. Das Lipoprotein (a) sank nach Rotweingenuss. Dies bestätigt einen günstigen Effekt der nicht alkoholischen Bestandteile von Rotwein, wahrscheinlich der Polyphenole.

\section{Akutwirkung von Krafttraining}

\section{Blutdruck sinkt bis zum frühen Morgen}

In dieser Untersuchung sollte die akute Wirkung einer Krafttrainingseinheit auf den Blutdruck von 13 Frauen geprüft werden, 8 mit Übergewicht und 5 mit Adipositas. Es gab zwei Gruppen: Eine sollte 30 Minuten im Sitzen ruhen, die andere drei Sätze Widerstandstraining mit 10 Wiederholungen bei $60 \%$ der Maximalkraft machen. Zudem wurde alle 10 Minuten bis eine Stunde nach dem Training der Ruheblutdruck sowie der 24-h-Blutdruck gemessen.

Tibana RA, Pereira GB, Navalta JW et al. Int J Sports Med 2012 Oct 12. [Epub ahead of print]
Ergebnisse: Der systolische Blutdruck war nach 10, 30 und 40 Minuten im Vergleich $\mathrm{zu}$ Ruhebedingungen und im Ver- gleich zur ruhenden Kontrollgruppe signifikant niedriger. Der diastolische Blutdruck sank zum Zeitpunkt 10 und 40 Minuten nach Belastung im Vergleich zur Kontrolle ebenfalls signifikant. Über Nacht war der Blutdruck um 4,2/4,1 mmHg gesenkt. Im 24-Std.-Mittel war der Blutdruck nach Krafttraining um 3,6 und 4,1 mmHg im Vergleich zur Kontrolle abgesenkt. Dies lässt den Autoren zufolge darauf schließen, dass ein Krafttraining den zirkadianen Rhythmus des Blutdrucks akut günstig beeinflusst, und zwar v.a. in den Nacht- und Morgenstunden, die als kritische Zeitabschnitte mit einem erhöhten kardiovaskulären Risiko gelten.

Quelle (3 Texte): www.lifestyle-telegramm.de

\section{Es stählt Körper, aber schützt es auch Herzen?}

Ein individuell gestaltetes und überwachtes Ganzkörper-Krafttraining mit moderater bis submaximaler Intensität bei Kindern scheint gesundheitlich unbedenklich zu sein und hat im Trend günstige Effekte auf die Körperzusammensetzung. Ein Einfluss auf kardiovaskuläre Risikofaktoren kann aber nicht bestätigt werden. Für diese Übersichtsarbeit wurden systematisch Interventionsstudien gesichtet, die nur den Einsatz von Krafttraining bei übergewichtigen und adipösen Kindern im Alter von 3 bis 18 Jahren geprüft und die Kör-

Dietz P, Hoffmann S, Lachtermann E, Simon P. Obes Facts. 2012;5(4):546-60. perzusammensetzung und kardiovaskuläre Risikofaktoren als Endpunkte einbezogen hatten.
Ergebnisse: Nur 6 Studien genügten den Aufnahmekriterien. Die Durchhalterate lag bei $84 \%$. In vier Studien gab es eine signifikante Veränderung der Körperzusammensetzung, d.h. einen Anstieg der fettfreien Körpermasse und des BMI bei gleichzeitiger Abnahme der Körperfettmasse. In drei Studien hatte man den Effekt von Krafttraining auf kardiovaskuläre Risikofaktoren gemessen. Nur in einer Studie fand man eine signifikante Senkung des systolischen Blutdrucks.

In zukünftigen Studien sollte der Einfluss von Krafttraining auf kardiovaskuläre Risikofaktoren bei übergewichtigen und adipösen Kindern mit größeren Teilnehmerzahlen überprüft und dabei sowohl Krafttraining allein als auch in Kombination mit Ernährungsumstellungen anwendet werden. 